\title{
A Nanodiamond-Based Theranostic Agent for Light-Controlled Intracellular Heating and Nanoscale Temperature Sensing
}

Yingke Wu, $\uparrow$ Md Noor A Alam, $\uparrow$ Priyadharshini Balasubramanian, Anna Ermakova, Stephan Fischer, Holger Barth, Manfred Wagner, Marco Raabe, * Fedor Jelezko* and Tanja Weil*

Y. Wu, M. N. A. Alam, Dr. A. Ermakova, Dr. M. Wagner, M. Raabe, Prof. Dr. T. Weil

Max Planck Institute for Polymer Research, Ackermannweg 10, 55128 Mainz, Germany

*E-mail: weil@mpip-mainz.mpg.de, raabe@mpip-mainz.mpg.de

M. N. A. Alam, M. Raabe, Prof. Dr. T. Weil

Institute of Inorganic Chemistry I, Ulm University, Albert-Einstein-Allee 11, 89081 Ulm, Germany

P. Balasubramanian, Prof. Dr. F. Jelezko

Institute for Quantum Optics, Ulm University, Albert-Einstein-Allee 11, 89081 Ulm, Germany *E-mail: fedor.jelezko@uni-ulm.de

Dr. S. Fischer, Prof. Dr. H. Barth

Institute of Pharmacology and Toxicology, Ulm University Medical Center, Albert-EinsteinAllee 11, 89081 Ulm, Germany

Keywords: Nanodiamond, nanogel, intracellular temperature manipulation and sensing, photothermal application.

\footnotetext{
Abstract

Temperature is an essential factor in all biological processes and most of the biological activities are related to temperature. However, information about the temperature in living cells are limited. In photothermal therapy (PTT), an emerging cancer treatment due to its high spatial control and non-invasive nature, the local effect of intracellular temperature change is not well understood. Many highly efficient photothermal agents have been developed in the past
} 
decades. However, almost none of them have investigated the intracellular change in temperature, for which highly sensitive and sophisticated nanothermometers are required which should not be affected by intracellular factors including $\mathrm{pH}$ or ions. Fluorescent nanodiamonds (ND) are a unique carbon material possessing optical defects inside the carbon lattice that enable sensing of temperature on a nanoscale completely independent of external conditions. Herein, we coat ND with polymers to form a nanogel shell around NDs that can absorb indocyanine green, a common photothermal agent, to obtain nanodiamond-nanogelindocyanine green. Upon irradiation, we not only show successful killing of cancer cells with a high control in space but we are also able to sense the increase in temperature using single NDs as nanothermometers in cells. This approach pushes the PTT research to an intracellular level and opens a door to explore the influence of the intracellular temperature on biological processes in living cells.

\section{Introduction}

Temperature plays a fundamental role in every biological process in living organisms. Most biological activities are temperature-controlled or temperature-related, for instance cell differentiation, proliferation, and death ${ }^{1}$, protein function ${ }^{2,3}$ as well as gene expression. ${ }^{4}$ All these biological actions take place or are controlled at specific intracellular locations. Hence, probing or even manipulating the local intracellular temperature is important to understand the fundamental relationship between biological activities and their temperature, such as the precise monitoring of temperature during hyperthermia.

For example, several research groups have reported that tumor cells could be effectively killed if the temperature in cells exceeded $42^{\circ} \mathrm{C} .{ }^{5,6}$ To effectively destroy the tumors, the tumor core is often exposed to much higher temperatures (above $50{ }^{\circ} \mathrm{C}$ ) to ensure that the tumor edges also 
reach the required therapeutic temperature. ${ }^{7-9}$ Counterintuitively, mitochondria maintain a physiological temperature of close to $50{ }^{\circ} \mathrm{C}$ without harming the cell. ${ }^{10}$ Therefore, it is essential to gain a deeper understanding of the local intracellular temperature change and its effect on tumor cells in more detail.

This information would be very beneficial to optimize existing techniques such as photothermal therapy (PTT). PTT is routinely applied to induce temperature increase in diseased cells or tissues and shows a high potential in the field of cancer treatment. ${ }^{5,11}$ During PTT, a photothermal agent (PA) is delivered into tumor cells or tissue and upon illumination, the PA converts absorbed light energy into heat. Over time, this process leads to either partial or complete ablation of the tumor cells or tissue. ${ }^{12}$ PTT is non-invasive, has a strong promise to improve the recovery time, and provides a better output as a cancer treatment ${ }^{13}$. PAs are extremely important for the efficiency of PTT because it dependents on the accumulation of photo-responsive PA in the target region and its light-to-heat conversion efficiency. Welldesigned PAs should generate certain amount of heat after the absorption of non-toxic light, normally in the near-infrared range (NIR, 650-900 nm) to achieve a better penetration depth. ${ }^{14,15}$ During the past years, various PA have been designed, mainly in the form of nanomaterials, which benefit from the enhanced permeability and retention (EPR) effect after intravenous injection ${ }^{16,17}$. These nanomaterials include metal-based nanomaterials, ${ }^{18-22}$ carbonbased nanomaterials, ${ }^{23-28}$ and organic molecules-based nanomaterials. ${ }^{29,30}$ However, these PAs lack the function to sense the temperature. Therefore, it was not possible to correlate between local temperature and the effect on the cell.

To directly measure the intracellular temperature on nanoscale, different kinds of fluorescencebased thermometer, such as quantum $\operatorname{dots}^{31}$, metal-based complexes, polymers ${ }^{32}$, and genetically encoded proteins ${ }^{33}$ have been explored. However, biological factors like $\mathrm{pH}$, ion concentration, and/or intracellular viscosity could have an influence on the sensitivity of 
fluorescence-based thermometers, resulting in inaccurate readout of the temperature. Nanodiamonds (NDs), containing nitrogen-vacancy $\left(\mathrm{NV}^{-}\right)$centers, are highly bio-compatible and physicochemically inert and provide a fluorescence that shows neither photoblinking nor photobleaching. Furthermore, their fluorescence are hardly influenced by $\mathrm{pH}$, ion concentration, viscosity, molecular interaction, or organic solvent. ${ }^{34}$. In addition, the temperature response from NDs differs strongly from most fluorescence probes. Upon a change in temperature, the ground state spin levels of $\mathrm{NV}^{-}$centers in NDs are shifted..$^{35}$ Additionally, $\mathrm{NV}^{-}$centers are useful as highly sensitive nanoscale thermometers based on the thermal shift of the zero field splitting $\left(D_{0}\right)$ at $2.87 \mathrm{GHz}\left(\mathrm{m}_{\mathrm{s}}=0 \text { to } \mathrm{m}_{\mathrm{s}}= \pm 1\right)^{36-38}$ or the zero-phonon line (ZPL) at $637 \mathrm{~nm} .{ }^{39,40}$ Therefore, NDs are an ideal material for designing an intracellular selfreporting photothermal system to probe the local temperature change in a PTT process.

Herein, we report the preparation of a self-reporting photothermal system, called nanodiamondnanogel-indocyanine green (ND-NG-ICG). A schematic outline of the preparation of ND-NGICG is displayed in Figure 1A. The nanodiamond-nanogel (ND-NG) was prepared according to our previous work. ${ }^{41}$ Explicitly, hyperbranched polyethyleneimine (PEI) was used to precoated NDs in the presence of polyvinylpyrrolidone (PVP) as stabilizer and subsequently, a 4-arm polyethyleneglycol NHS ester was added to cross-link the precoated PEI on the surface of NDs in phosphate-buffered saline (PBS) buffer to form the nanogel shell. After purification, the positively charged ND-NG was obtained. Finally, anionic indocyanine green (ICG) was mixed with ND-NG solution to form ND-NG-ICG by strong electrostatic interaction. Furthermore, the photothermal effect and the temperature sensing were proven in vitro. 


\section{Results and discussion}

ND-NG was prepared as reported previously ${ }^{41}$ and was mixed with ICG. Afterwards, free ICG was removed by centrifugation to obtain pure ND-NG-ICG. The amount of ICG loaded on NDNG by electrostatic interactions is essential for the efficiency of PTT and was determined by the characteristic absorbance of ICG at $789 \mathrm{~nm}$. Considering that NDs could interfere during the absorbance measurement, we determined the concentration of free ICG which remained in the supernatant after centrifugation. We found that $64.5 \mu \mathrm{g}$ ICG was loaded on $200 \mu \mathrm{g}$ NDNG-ICG which is equal to a loading efficiency of $24.4 \%$ (Figure S1). Since ICG was loaded by non-covalent adsorption, which could cause unwanted leakage, we investigated the release of ICG over time. The ICG was release dramatically in the first 10 minutes and reached then the equilibrium. Only about $10 \%$ ICG was released over 20 days due to the strong interaction between ICG and ND-NG (Figure S2). ND-NG-ICG was characterized by dynamic light scattering (DLS; Figure 1B) and transmission electron microscopy (TEM; Figure 1C and 1D) to investigate its size distribution, shape, and morphology. ND-NG-ICG showed a monomodal size distribution in DLS (Figure 1B) with polydispersity index (PDI) of 0.243, and the hydrodynamic diameter increased from $37.7 \pm 0.23 \mathrm{~nm}$ of ND to $56.9 \pm 0.27 \mathrm{~nm}$ of ND-NG and finally, to $99.6 \pm 0.53 \mathrm{~nm}$ of ND-NG-ICG in Milli-Q water (Figure S3 ). These findings were supported by TEM images of ND-NG-ICG which did not show any obvious aggregation (Figure 1C).

The photophysical properties of NDs containing $\mathrm{NV}^{-}$centers are important for the application of ND-NG-ICG in intracellular temperature sensing. First, the emission spectra of ND and ICG as well as the absorption spectra of ICG were measured (Figure S3). We found that the emission spectra of ND and the absorption spectra of ICG overlap, which could induce an energy transfer upon irradiation of ND-NG-ICG at $532 \mathrm{~nm}$. To further check the influence of the coating on the $\mathrm{NV}^{-}$centers in NDs, ND-NG-ICG was dropped on a glass coverslip. Spectra measurements 
were performed on a custom-built confocal microscope using a $532 \mathrm{~nm}$ excitation with a power of $110 \mu \mathrm{W}$ in front of the objective (oil, NA $=1.35$ ). The spectra of ND-NG-ICG revealed that the intensity of the peak at $680 \mathrm{~nm}$ slightly decreased due to a low energy transfer to ICG. The zero phonon line of $\mathrm{NV}^{-}$at $637 \mathrm{~nm}$ was clearly visible without any background noise (Figure 1E). $\mathrm{NV}^{-}$centers in NDs are very sensitive to the surface charge and can switch to the dark state (positively charged NV center; $\mathrm{NV}^{+}$) under certain conditions. The results demonstrated that the $\mathrm{NV}^{-}$centers in ND-NG-ICG remained in the optically active state which was necessary for the intracellular temperature sensing. 


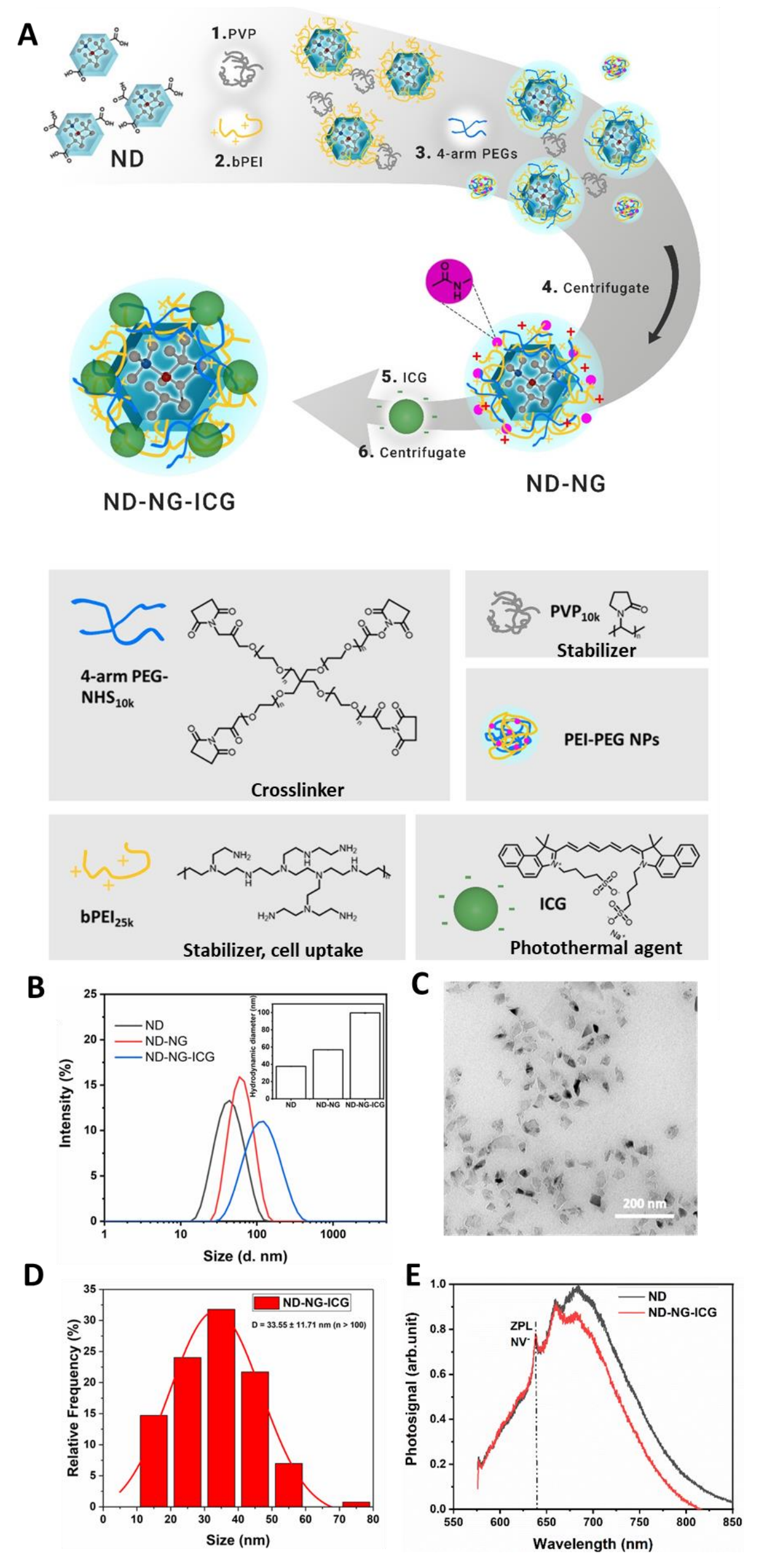


Figure 1. (A) Schematic illustration of the synthesis route of ND-NG-ICG. (B) The hydrodynamic diameter of ND, ND-NG, and ND-NG-ICG measured by DLS. (C) TEM images of ND-NG-ICG (scale bar = $200 \mathrm{~nm}$ ). (D) Histogram analysis of ND-NG-ICG. (E) Normalized emission spectra (ex. $532 \mathrm{~nm}$ ) of ND and ND-NG-ICG. The zero-phonon line (ZPL) of $\mathrm{NV}^{-}$is visible in both spectra.

To understand the influence of ND-NG-ICG on cells, first the biocompatibility of ND-NGICG was investigated in a human cervical carcinoma cell line (HeLa). As displayed in Figure 2A, ND-NG-ICG showed good cell viability after the treatment of the cells with a concentration up to $400 \mu \mathrm{g} / \mathrm{mL}$. In addition, as depicted in Figure 2B, ND-NG-ICG were efficiently taken up into HeLa cells, the cell could grow well, and the cell morphology was not altered. Moreover, to further investigate the location of ND-NG-ICG in cells, $10 \mu \mathrm{g} / \mathrm{mL}$ and $100 \mu \mathrm{g} / \mathrm{mL}$ ND-NGICG were incubated with HeLa cell for 4 hours, embedded in resin after stain, and cut to 100 $\mathrm{nm}$ section for TEM measurement (Figure 2C and 2D). We found that the ND-NG-ICGs can be internalized, encapsulated in a vesicle and taken in. The behavior was concentrationindependent. There is no significant difference of cell uptake between $10 \mu \mathrm{g} / \mathrm{mL}$ and 100 $\mu \mathrm{g} / \mathrm{mL}$ ND-NG-ICG. The only difference was that much less ND-NG-ICG were found in the cells for $10 \mu \mathrm{g} / \mathrm{mL}$ ND-NG-ICG. 
A

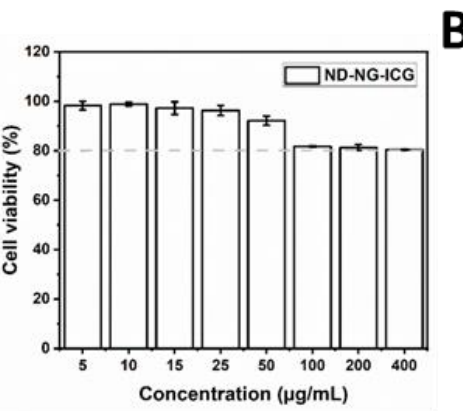

C

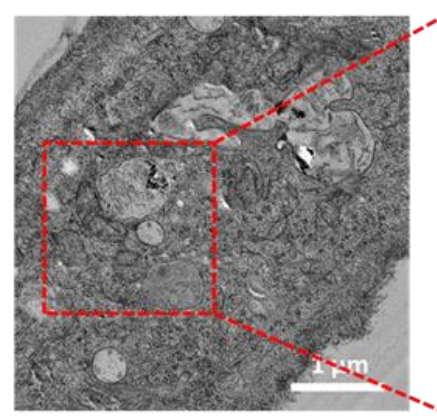

D

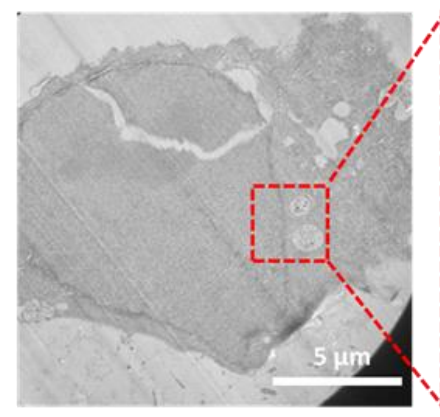

B
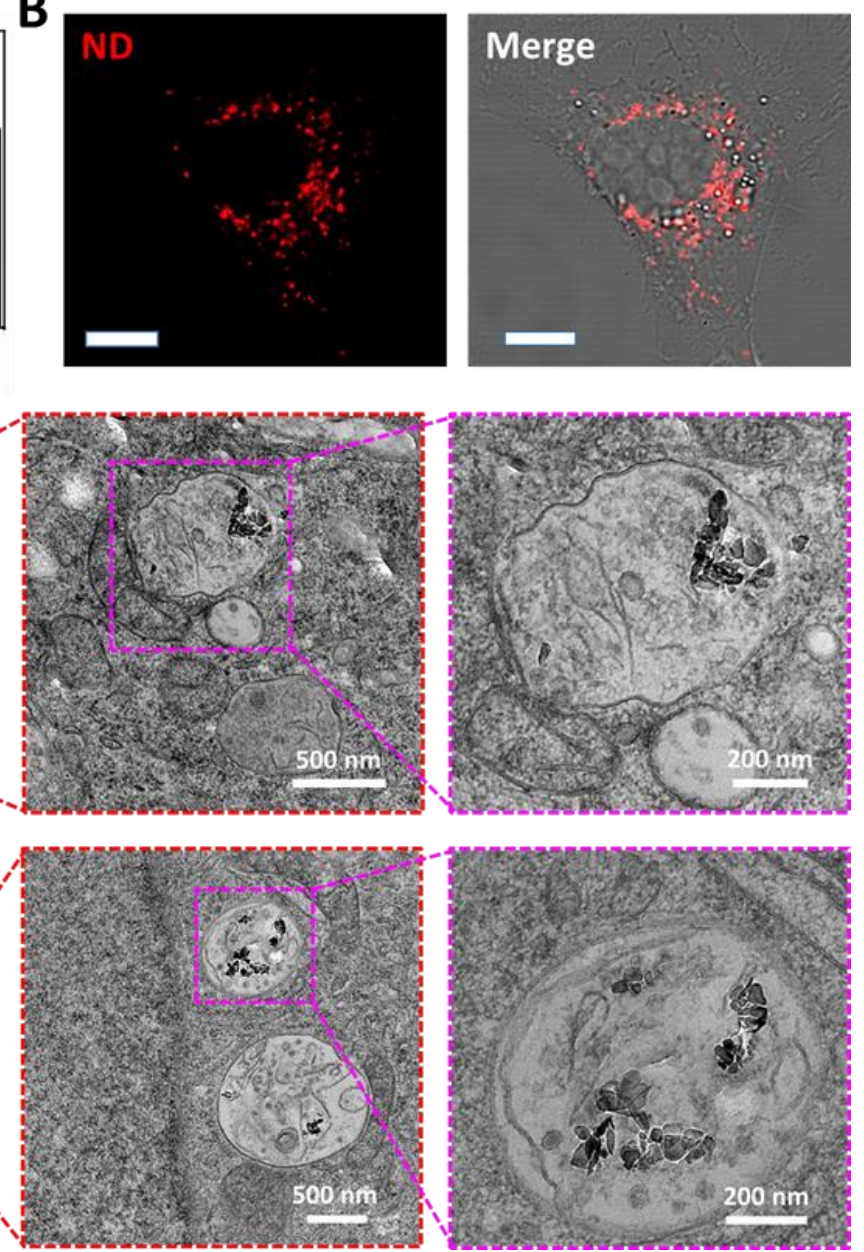

Figure 2. (A) Cell viability of HeLa cells after $4 \mathrm{~h}$ incubation with ND-NG-ICG. (B) Confocal microscopy images of ND-NG-ICG which were taken up into HeLa cells with a concentration of $100 \mu \mathrm{g} / \mathrm{mL}$ after 4 hours (scale bar $=10 \mu \mathrm{m}$ ). (C) TEM images of ND-NG-ICG which were taken up into HeLa cells with a concentration of $10 \mu \mathrm{g} / \mathrm{mL}$ after 4 hours. (D) TEM images of ND-NG-ICG which were taken up into HeLa cells with a concentration of $100 \mu \mathrm{g} / \mathrm{mL}$ after 4 hours.

To measure the nanoscale local temperature change in cells under irradiation, a home-built confocal fluorescence microscope combined with optically detected magnetic resonance (ODMR) spectroscopy was used (Figure 3A, Figure S5 and S6). Based on the physical property of $\mathrm{NV}^{-}$centers in $\mathrm{ND}$, the zero field splitting $D_{0}$ are changed in response to a change in temperature which provides a robust readout of temperature. As shown in Figure 3C, the 
ODMR spectra of ND-NG-ICG under NIR irradiation were recorded and fitted with a sum of double Lorentzuans; after 3 minutes irradiation, the ODMR spectrum was shifted to the low frequency. However, for the ND-NG samples (Figure S7), there is no significant shift in the ODMR spectra under NIR irradiation. To readout the intracellular temperature change, the ODMR spectra was recorded up to 7 minutes continuously and the data were processed every 60 seconds. The ODMR spectra were fitted with a double Lorentzian function, and the temperature change were extracted according to the equation:

$$
\Delta T=\frac{\Delta D}{\alpha}
$$

where $\Delta D$ is the shift in the transition frequency and $\alpha=d D / d T=-74 \mathrm{kHz} / \mathrm{K}$ is the temperature susceptibility. In addition, the irradiation time dependence of the temperature change curve was obtained and fitted (Figure 3E); it was found that the temperature increased sharply by more than $30{ }^{\circ} \mathrm{C}$ and saturated after approximately 250 seconds of irradiation for the ND-NG-ICG samples but there was no significant temperature change for the ND-NG samples under irradiation.

Moreover, we investigated the photothermal effect of ND-NG-ICG by thermocouple in solution. We quantified the photothermal effect of ND-NG-ICG measuring the change in temperature of water upon irradiation of $810 \mathrm{~nm}$ with the power of $0.35 \mathrm{~W} / \mathrm{cm}^{2}$. Firstly, we evaluated the change in temperature of ND-NG in absence of the ICG dye at different concentrations (Figure 3D). We found a concentration-independent temperature increase of less than $2{ }^{\circ} \mathrm{C}$ which reached a saturation after 5 minutes of irradiation. In contrast, ND-NGICG revealed a concentration-dependent increase of temperature of $2{ }^{\circ} \mathrm{C}$ to $18{ }^{\circ} \mathrm{C}$ for concentrations between $10 \mu \mathrm{g} / \mathrm{mL}$ and $200 \mu \mathrm{g} / \mathrm{mL}$ after 5 minutes of irradiation. The concentration-dependent increase is similar to free ICG. Free ICG at a concentration ranging from $10 \mu \mathrm{g} / \mathrm{mL}$ to $200 \mu \mathrm{g} / \mathrm{mL}$ (which equals a concentration ranging from $31 \mu \mathrm{g} / \mathrm{mL}$ to 620 
$\mu \mathrm{g} / \mathrm{mL}$ of ND-NG-ICG) showed an increase in temperature of $11^{\circ} \mathrm{C}$ to $36{ }^{\circ} \mathrm{C}$ after 5 minutes of irradiation (Figure S4A). Furthermore, the photothermal effect of equimolar free ICG and ND-NG-ICG is comparable (Figure S4C). These results indicated that the efficiency to produce heat is not impaired for ICG which was loaded on ND-NG.

The operating principle of thermometry using NV centers relies on the accurate measurement of the transition frequency, which it can be optically detected with high spatial resolution. For NV-based thermometry, the temperature sensitivity can be calculated the following equation:

$$
\eta_{E S R} \approx \frac{4}{3 \sqrt{3}} \frac{1}{d D / d T} \frac{\Delta f}{C \sqrt{R}}
$$

where $d D / d T$ is the NV temperature susceptibility, $\Delta f$ is the approximate ODMR linewidth, $C$ is ODMR contrast and $R$ is the average photon counts. Here $d D / d T=74 \mathrm{kHz} / \mathrm{K}, C \approx 0.05, \Delta f=$ $5 \mathrm{MHz}$ and $R=3 \mathrm{M}$ cts/s, then the calculated sensitivity is $\eta=600 \mathrm{mK} / \sqrt{\mathrm{Hz}}$. However, theoretically a single $\mathrm{NV}$ can potentially exhibit a sensitivity better than $1 \mathrm{mK} / \sqrt{ } \mathrm{Hz}$. 
A

\section{Nanoscale thermometer}
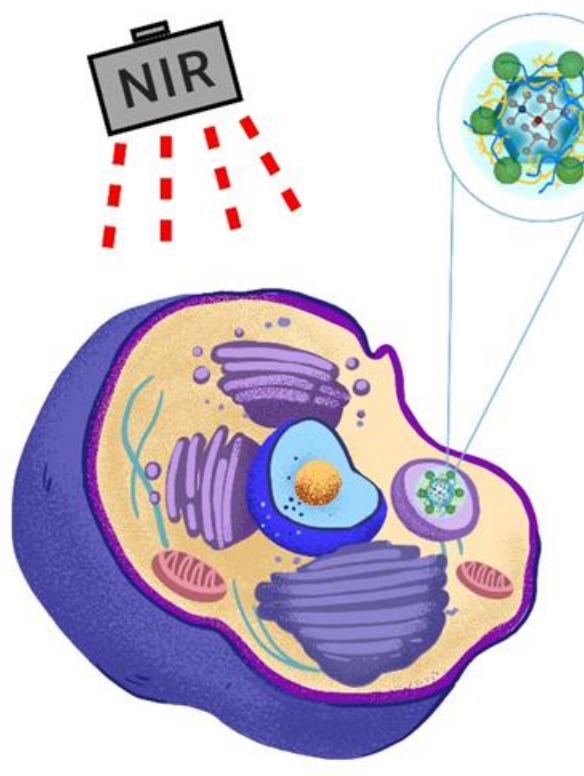

B

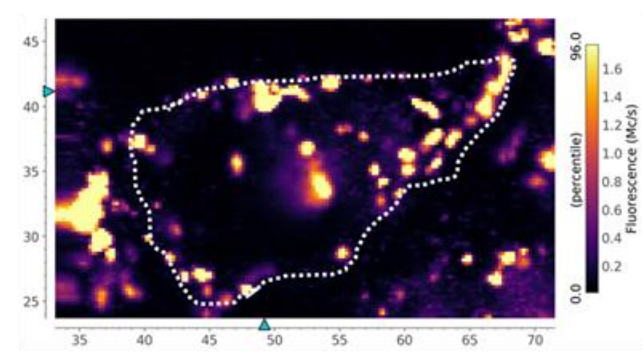

D

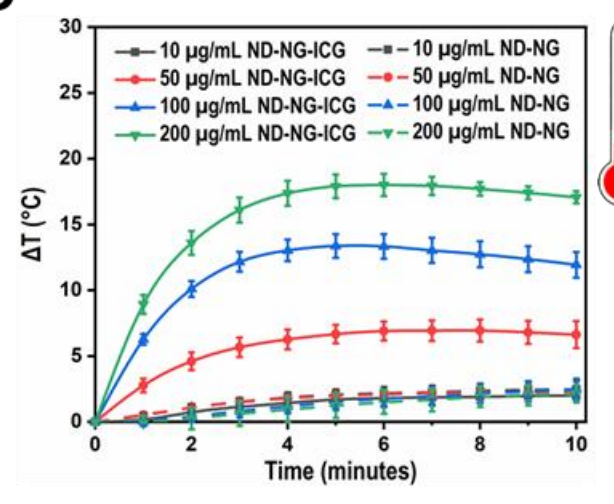

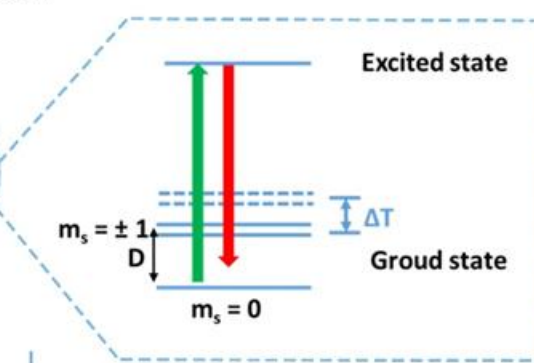

Readout

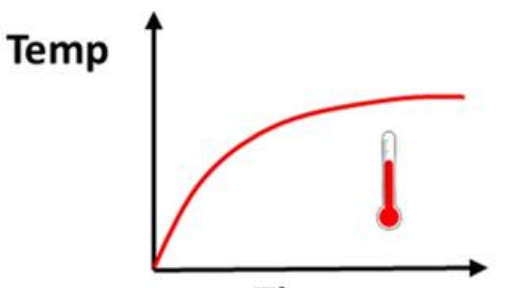

Time

C

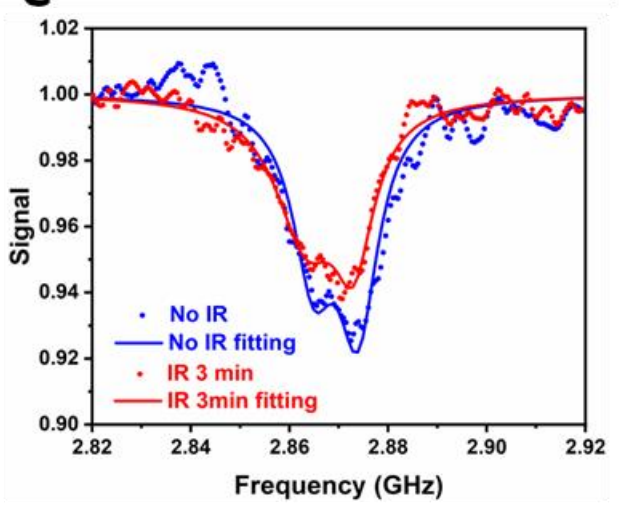

E

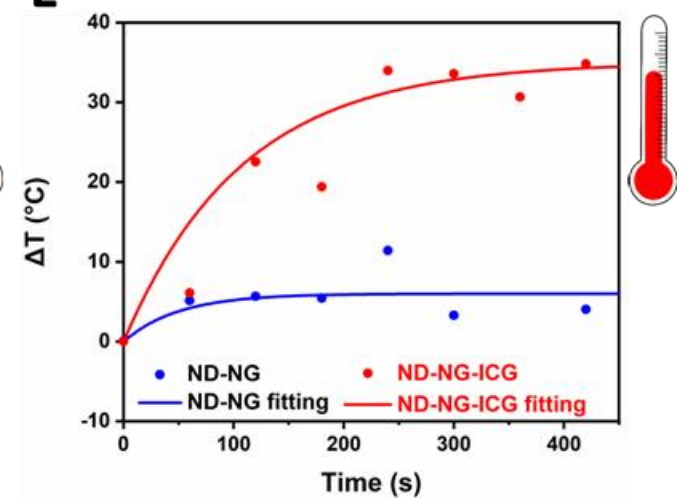

Figure 3. (A) Schematic presentation of the temperature measurement in living cells. Simplified energy levels diagram of the $\mathrm{NV}^{-}$center in nanodiamond displaying a ground state of spin triplet and an excited state. Under zero magnetic field, the $\mathrm{m}_{\mathrm{s}}= \pm 1$ sub-levels are split 
from the $\mathrm{m}_{\mathrm{s}}=0$ state by a temperature-dependent zero field splitting $D_{0}$. The intracellular temperature change can be monitored by the shift of the zero-field splitting parameter $D_{0}$ using the ODMR technique. At room temperature, $D_{0} \approx 2.87 \mathrm{GHz}$ and it varies depending on temperature $\mathrm{T}$ as $\Delta T=\Delta D / \alpha$, where $\alpha=-74 \mathrm{kHz} / \mathrm{K}$. (B) Fluorescence image of ND-NG-ICG in a living cell. (C) Representative ODMR spectra of ND-NG-ICG which was fitted with a sum of double Lorentzians, under near-infrared (NIR) irradiation (810 nm lamp; $\left.0.35 \mathrm{~W} / \mathrm{cm}^{2}\right)$ at 0 minute and 3 minutes. (D) The thermal profile of ND-NG and ND-NG-ICG with a concentration of $10 \mu \mathrm{g} / \mathrm{mL}, 50 \mu \mathrm{g} / \mathrm{mL}, 100 \mu \mathrm{g} / \mathrm{mL}$, and $200 \mu \mathrm{g} / \mathrm{mL}$ under near-infrared (NIR) irradiation $\left(810 \mathrm{~nm}\right.$ lamp; $\left.0.35 \mathrm{~W} / \mathrm{cm}^{2}\right)$. (E) The change of intracellular temperature measured by ODMR for NG-NG-ICG and ND-NG over 420 seconds under near-infrared (NIR) irradiation (810 $\mathrm{nm}$ lamp; $\left.0.35 \mathrm{~W} / \mathrm{cm}^{2}\right)$.

To understand the photothermal effect on cells, a LED light with a wavelength of $810 \mathrm{~nm}$ was focused onto the sample area through the objective of a microscope for 20 minutes. The light power density was $0.35 \mathrm{~W} / \mathrm{cm}^{2}$, which is the same value we used in ODMR measurements later on. After 20 minutes of irradiation, the cells were further incubated for $4 \mathrm{~h}$ and live/dead staining of PTT treated cells was performed. As shown in Figure 4B, without irradiation at 810 $\mathrm{nm}$, cells could proliferate well, showed a normal cell morphology, and almost no dead cells were found neither at $10 \mu \mathrm{g} / \mathrm{mL}$ of ND-NG-ICG nor at $100 \mu \mathrm{g} / \mathrm{mL}$ of ND-NG-ICG. After irradiation at $810 \mathrm{~nm}$, the cell viability was not impaired at low concentrations of ND-NG-ICG $(10 \mu \mathrm{g} / \mathrm{mL}$ ) and ND-NG (Figure S8). However, most of the cells treated with a high concentration of ND-NG-ICG $(100 \mu \mathrm{g} / \mathrm{mL})$ were dead in the live/dead imaging within the irradiated area.

According to the ODMR measurement, the local temperature close to the surface of ND-NGICG is above $50{ }^{\circ} \mathrm{C}$ after 250 seconds of irradiation and the local temperature is independent of the concentration because both $10 \mu \mathrm{g} / \mathrm{mL}$ and $100 \mu \mathrm{g} / \mathrm{mL}$ revealed a similar local 
temperature increase. This data revealed that cells can compensate a local high temperature (> $50{ }^{\circ} \mathrm{C}$ ) for a low concentration of ND-NG-ICG but a high concentration of ND-ND-ICG resulted in cell death probably due to an accumulative effect of the local temperature spots. Our results also support that small areas in cells can possess different temperatures without effecting the cell viability. For example, it is reported that in mitochondria the temperature is higher than in the cytosol. ${ }^{10}$

\section{A}

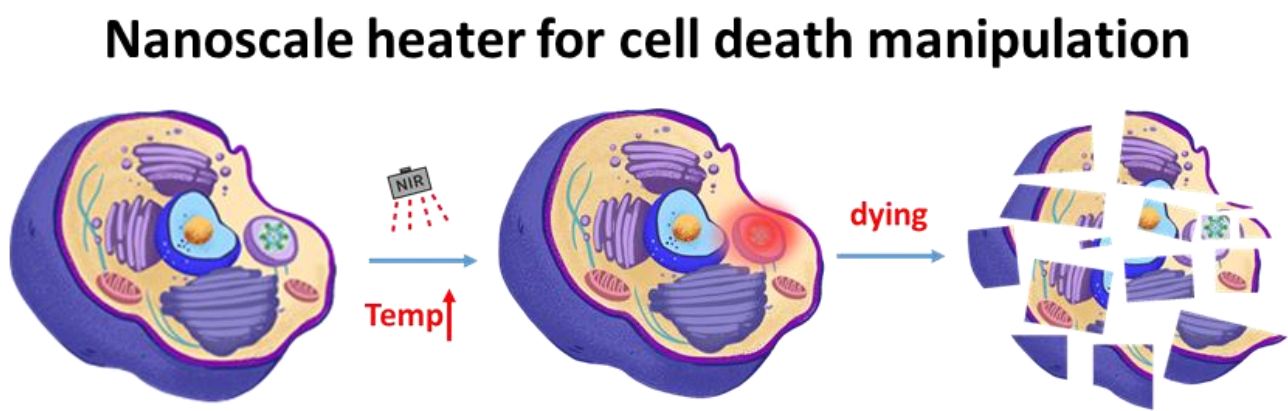

B

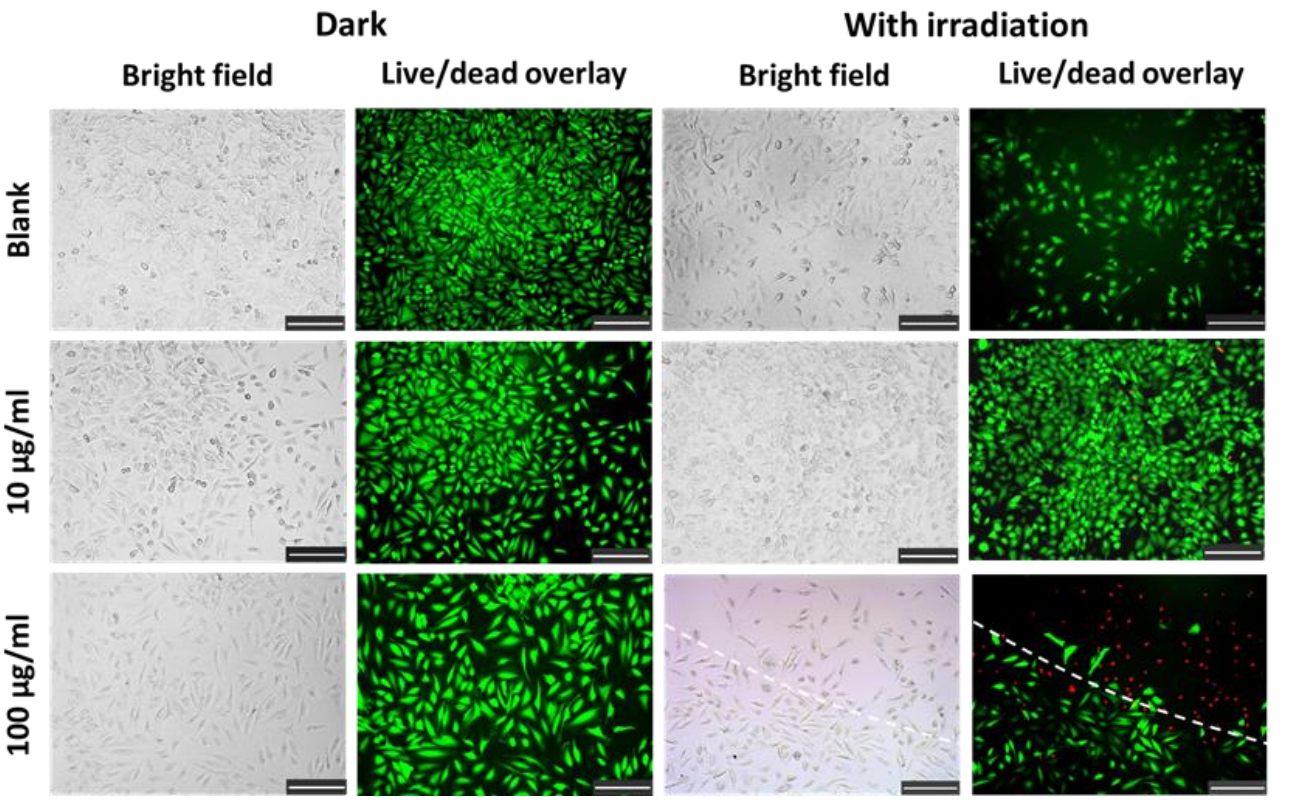

Figure 4. (A) Sketch of nanoscale heater for cell death manipulation. (B) Live/dead staining of HeLa cells incubated with different concentration of ND-NG-ICG after 20 minutes irradiation using a near-infrared (NIR) LED lamp (810 nm lamp; $0.35 \mathrm{~W} / \mathrm{cm}^{2}$; scale bar $\left.=200 \mu \mathrm{m}\right)$. Green and red color represent live and dead cells respectively. 
In summary, we have successfully prepared a nanoscale photothermal agent equipped with a self-reporting system. This agent monitored the temperature increase due to the photothermal effects in situ and in living cells. The ODMR results showed that the local temperature increased by $30{ }^{\circ} \mathrm{C}$ within 250 seconds. Moreover, we found that the cells can tolerate a high local temperature of more than $50{ }^{\circ} \mathrm{C}$ if the concentration of the photothermal agent is low. This data support that the intracellular temperature of cells can be inhomogeneous and can even differ by $30{ }^{\circ} \mathrm{C}$ without affecting the cell viability. Our study investigated the local temperature change induced by a photothermal agent, which has not been revealed by former studies on PTT. This work helps to gain a deeper understanding of the photothermal effect in nanoscale and opens the way for further studies on photothermal effect in different intracellular organelles and its effect on cells. Furthermore, it has been reported that a local temperature change can influence the cell division time $^{42}$ and the differentiation of cells. ${ }^{43}$ A ND-based nanothermometer can help to investigate temperature-driven biological processes in more detail.

\section{Conflicts of interest}

The authors declare no competing financial interest.

\section{Supporting Information}

Supporting Information is available from the $* * * * *$ or from the author.

\section{Acknowledgements}

The authors thank Mr. Kai Philipps for the ICG emission spectrum measurement, Christoph Sieber for the preparation TEM samples of cells, Dr. David Yuen Wah Ng for the fruitful 
discussion and suggestions. The authors are grateful for the financial support from the European Union's Horizon 2020 Research and Innovation Program under FETOPEN grant agreement no. 858149 (AlternativeToGd) and from the Deutsche Forschungsgemeinschaft (DFG, German Research Foundation) - Project number 316249678 - SFB 1279 (C04). Y. Wu thanks the China Scholarship Council for a fellowship.

\section{Author Contributions}

†Yingke Wu and Md Noor A Alam contributed equally.

\section{References}

1 Franceschi, C. Cell proliferation, cell death and aging. Aging Clin. Exp. Res. 1, 3-15 (1989).

2 Marsden, H., Crombie, I. K. \& Subak-Sharpe, J. Control of protein synthesis in herpesvirusinfected cells: analysis of the polypeptides induced by wild type and sixteen temperaturesensitive mutants of HSV strain 17. J. Gen. Virol. 31, 347-372 (1976).

3 Martinez, J., Georgoff, I. \& Levine, A. Cellular localization and cell cycle regulation by a temperature-sensitive p53 protein. Genes Dev. 5, 151-159 (1991).

4 Seymour, R. S. Biophysics and physiology of temperature regulation in thermogenic flowers. Biosci. Rep. 21, 223-236 (2001).

5 Wei, W., Zhang, X., Zhang, S., Wei, G. \& Su, Z. Biomedical and bioactive engineered nanomaterials for targeted tumor photothermal therapy: a review. Mater. Sci. Eng.C 104, 109891 (2019).

6 Doughty, A. C. et al. Nanomaterial applications in photothermal therapy for cancer. Materials 12, 779 (2019).

7 Hsiao, C.-W. et al. Photothermal tumor ablation in mice with repeated therapy sessions using NIR-absorbing micellar hydrogels formed in situ. Biomaterials 56, 26-35 (2015).

8 Chen, Q. et al. Photothermal therapy with immune-adjuvant nanoparticles together with checkpoint blockade for effective cancer immunotherapy. Nat. Commun. 7, 1-13 (2016).

9 Zhang, C. et al. A polyoxometalate cluster paradigm with self-adaptive electronic structure for acidity/reducibility-specific photothermal conversion. J. Am. Chem. Soc. 138, 8156-8164 (2016).

10 Chrétien, D. et al. Mitochondria are physiologically maintained at close to 50 C. PLoS Biol. 16, e2003992 (2018).

11 Chen, F. \& Cai, W. Nanomedicine for targeted photothermal cancer therapy: where are we now? Nanomed. 10, 1-3 (2015).

12 Norouzi, H., Khoshgard, K. \& Akbarzadeh, F. In vitro outlook of gold nanoparticles in photothermal therapy: a literature review. Lasers Med. Sci. 33, 917-926 (2018).

13 Espinosa, A. et al. Cancer cell internalization of gold nanostars impacts their photothermal efficiency in vitro and in vivo: toward a plasmonic thermal fingerprint in tumoral environment. Adv. Healthcare Mater. 5, 1040-1048 (2016).

14 Huang, X., El-Sayed, I. H., Qian, W. \& El-Sayed, M. A. Cancer cell imaging and photothermal therapy in the near-infrared region by using gold nanorods. J. Am. Chem. Soc. 128, 21152120 (2006). 
Tchounwou, C. et al. Hybrid theranostic platform for second near-IR window light triggered selective two-photon imaging and photothermal killing of targeted melanoma cells. ACS Appl. Mater. Interfaces 7, 20649-20656 (2015).

Matsumura, Y. \& Maeda, H. A new concept for macromolecular therapeutics in cancer chemotherapy: mechanism of tumoritropic accumulation of proteins and the antitumor agent smancs. Cancer Res. 46, 6387-6392 (1986).

17 Jaque, D. et al. Nanoparticles for photothermal therapies. Nanoscale 6, 9494-9530 (2014).

18 Tsai, M.-F. et al. Au nanorod design as light-absorber in the first and second biological nearinfrared windows for in vivo photothermal therapy. ACS Nano 7, 5330-5342 (2013).

19 Loo, C. et al. Nanoshell-enabled photonics-based imaging and therapy of cancer. Technol. Cancer Res. Treat. 3, 33-40 (2004).

Sun, Y., Mayers, B. T. \& Xia, Y. Template-engaged replacement reaction: a one-step approach to the large-scale synthesis of metal nanostructures with hollow interiors. Nano Lett. 2, 481485 (2002).

21 Huang, X. et al. Freestanding palladium nanosheets with plasmonic and catalytic properties. Nat. Nanotechnol. 6, 28-32 (2011).

22 Li, Y., Lu, W., Huang, Q., Li, C. \& Chen, W. Copper sulfide nanoparticles for photothermal ablation of tumor cells. Nanomed. 5, 1161-1171 (2010).

23 Geng, B. et al. NIR-responsive carbon dots for efficient photothermal cancer therapy at low power densities. Carbon 134, 153-162 (2018).

24 Liang, C. et al. Tumor metastasis inhibition by imaging - guided photothermal therapy with single - walled carbon nanotubes. Adv. Mater. 26, 5646-5652 (2014). Yang, K. et al. The influence of surface chemistry and size of nanoscale graphene oxide on photothermal therapy of cancer using ultra-low laser power. Biomaterials 33, 2206-2214 (2012).

26 Yang, K. et al. Graphene in mice: ultrahigh in vivo tumor uptake and efficient photothermal therapy. Nano Lett. 10, 3318-3323 (2010).

27 Tian, B., Wang, C., Zhang, S., Feng, L. \& Liu, Z. Photothermally enhanced photodynamic therapy delivered by nano-graphene oxide. ACS Nano 5, 7000-7009 (2011).

28 Ryu, T. K., Baek, S. W., Kang, R. H. \& Choi, S. W. Selective photothermal tumor therapy using nanodiamond - based nanoclusters with folic acid. Adv. Funct. Mater. 26, 6428-6436 (2016). Yu, J., Yaseen, M. A., Anvari, B. \& Wong, M. S. Synthesis of near-infrared-absorbing nanoparticle-assembled capsules. Chem. Mater. 19, 1277-1284 (2007).

30 Liu, Y., Song, N., Li, Z., Chen, L. \& Xie, Z. Near-infrared nanoparticles based on aza-BDP for photodynamic and photothermal therapy. Dyes Pigm. 160, 71-78 (2019).

31 Yang, J.-M., Yang, H. \& Lin, L. Quantum dot nano thermometers reveal heterogeneous local thermogenesis in living cells. ACS Nano 5, 5067-5071 (2011).

32 Okabe, K. et al. Intracellular temperature mapping with a fluorescent polymeric thermometer and fluorescence lifetime imaging microscopy. Nat. Commun. 3, 1-9 (2012). Kiyonaka, S. et al. Genetically encoded fluorescent thermosensors visualize subcellular thermoregulation in living cells. Nat. Methods 10, 1232-1238 (2013).

34 Sekiguchi, T., Sotoma, S. \& Harada, Y. Fluorescent nanodiamonds as a robust temperature sensor inside a single cell. Biophys. Physicobiol. 15, 229-234 (2018). Acosta, V. M. et al. Temperature dependence of the nitrogen-vacancy magnetic resonance in diamond. Phys. Rev. Lett. 104, 070801 (2010).

36 Neumann, P. et al. High-precision nanoscale temperature sensing using single defects in diamond. Nano Lett. 13, 2738-2742 (2013).

37 Kucsko, G. et al. Nanometre-scale thermometry in a living cell. Nature 500, 54-58 (2013).

38 Simpson, D. A. et al. Non-neurotoxic nanodiamond probes for intraneuronal temperature mapping. ACS Nano 11, 12077-12086 (2017). 
39 Plakhotnik, T., Doherty, M. W., Cole, J. H., Chapman, R. \& Manson, N. B. All-optical thermometry and thermal properties of the optically detected spin resonances of the NVcenter in nanodiamond. Nano Lett. 14, 4989-4996 (2014).

40 Tsai, P. C. et al. Measuring nanoscale thermostability of cell membranes with single golddiamond nanohybrids. Angew. Chem. Int. Ed. 56, 3025-3030 (2017).

$41 \mathrm{Wu}, \mathrm{Y}$. et al. Fluorescent Nanodiamond-Nanogels for Nanoscale Sensing and Photodynamic Applications. https://doi.org/10.26434/chemrxiv.13317494.v2.

42 Choi, J. et al. Probing and manipulating embryogenesis via nanoscale thermometry and temperature control. Proc. Natl. Acad. Sci. U. S. A.117 14636-14641 (2020).

43 Yoon, H.-H., Han, M.-J., Park, J.-K., Lee, J.-H. \& Seo, Y.-K. Effect of low temperature on Schwann-like cell differentiation of bone marrow mesenchymal stem cells. Tissue Eng. Regener. Med. 12, 259-267 (2015). 